\title{
New therapeutic options for bile acid malabsorption diarrhea
}

\author{
Alejandro Valencia-Rodríguez ${ }^{1}$, Jorge Aquino-Matus ${ }^{1}$, Alfonso Vera-Barajas ${ }^{1}$, Xingshun $\mathbf{Q i}^{2}$, \\ Nahum Méndez-Sánchez ${ }^{1,3}$ \\ ${ }^{1}$ Liver Research Unit, Medica Sur Clinic and Foundation, Mexico City, Mexico; ${ }^{2}$ Department of Gastroenterology, General Hospital of Northern \\ Theater Command, Shenyang 110840, China; ${ }^{3}$ Faculty of Medicine, National Autonomous University of Mexico, Mexico City, Mexico \\ Correspondence to: Nahum Méndez-Sánchez, MD, MSc, PhD. Liver Research Unit, Medica Sur Clinic and Foundation, Puente de Piedra 150, Col. \\ Toriello Guerra, ZP. 14050, Mexico City, Mexico. Email: nmendez@medicasur.org.mx; nah@unam.mx.
}

Submitted Aug 30, 2019. Accepted for publication Sep 16, 2019.

doi: 10.21037/atm.2019.09.112

View this article at: http://dx.doi.org/10.21037/atm.2019.09.112

We have read with great interest the work published recently by Kårhus et al. (1) where they describe the efficacy of the glucagon-like peptide-1 (GLP-1) agonist Liraglutide in two patients diagnosed with severe bile acid malabsorption (BAM) by causing a complete remission of symptoms.

In 1972, Hofmann and Poley (2) studied 9 patients with intestinal resection and bile acid diarrhea (BAD) to define the importance of BAM in the pathogenesis of chronic diarrhea in this type of patients, as well as the benefit shown using cholestyramine and dietary changes. They found that resections of $<1$ meter responded well to treatment with bile acid sequestrants (BAS) while those who had resections of $>1$ meter did not have benefits with the use of BAS presenting even more episodes of diarrhea and steatorrhea, but they responded well to dietary changes by replacing long-chain triglycerides with medium-chain triglycerides.

It is currently known that BAD is one of the most frequent causes associated with chronic diarrhea (diarrhea $>4$ weeks). It is estimated that in developed countries $5 \%$ of the population has chronic diarrhea, and approximately $25-33 \%$ are secondary to BAD (3). Globally, a prevalence of BAD of $1 \%$ is estimated (4). For its classification BAM has been divided into 3 types (5): in type 1 (secondary to an ileal dysfunction) it mainly includes patients with Crohn's disease and/or intestinal resection. The mechanism involved is an alteration in the absorption of bile acids (BAs). In type 2 (idiopathic) it generally occurs in patients with irritable bowel disease (IBD), the mechanisms predominantly involved in this type are alterations in gastrointestinal (GI) motility and intestinal dysbiosis in post-infectious diarrhea. In type 3 (secondary to GI disorders not associated with ileal dysfunction) there are included patients with chronic pancreatitis where alterations in the secretion of bicarbonate coexist, in celiac disease where atrophy of the intestinal mucosa is seen, in postvagotomy patients where an increase in GI motility is associated and in postcholecystectomy patients due to impaired enterohepatic circulation. Regardless the clinical spectrum of BAM, in all 3 types there is an alteration in the transport and receptors of BAs in the intestine due to a modification of the BAs pool (5) (Figure 1).

Despite being a frequent condition among patients with chronic diarrhea, it is still an underdiagnosed condition. Different methods have been used to try to diagnose BAM, the best method currently described is the 75 -selenium homocholic acid taurine (SeHCAT) test. It is a nuclear medicine investigation considered to be the gold standard for the diagnosis of BAM by providing a quantitative assessment to estimate the severity and the possible response to therapy (6). From the work of Hoffman and Poley, the BAS (cholestyramine, colestipol and colesevelam) were considered the treatment of choice for this disease although its therapeutic success has been questioned. BAS are polymeric resins with the ability to bind to BAs in the small intestine, reducing their absorption and increasing their elimination through feces. This causes a de novo synthesis at the liver and a decrease in plasmatic LDL-C concentration (7). BAS are also involved in triglyceride metabolism by increasing plasmatic VLDL concentrations, this effect is partly mediated by Farnesoid X receptor (FXR), considered as the main nuclear receptor that regulates BAs synthesis and other compounds such as glucose, lipids and aminoacids (7). The treatment with BAS changes the BAs 
pool making them less effective to activate FXR (8).

Furthermore, in the last decade it has been seen suggested that BAS influence glucose metabolism by changing the BAs pool obtained with the treatment of these drugs which can activate with greater affinity the $G$ proteincoupled bile acid receptor 1 (TGR5). This receptor is involved in the metabolism of BAs in an independent way of FXR and fibroblast growth factor-19 (FGF-19). Once BAs activate TGR5, internalization of the receptor is promoted by increasing intracellular levels of cAMP and activation of protein kinase A stimulating the intestine $\mathrm{L}$ cells to release GLP-1. This theory is supported by an experimental study carried out in rats with type 2 diabetes mellitus (T2DM) which received treatment with BAS, resulting in an increase in the expression of GLP-1, YY peptide and insulin release compared with the control group (9). In addition, in another clinical study with patients with T2DM treated with BAS, a postprandial increase in GLP-1 was seen at 2 hours (10).

In this case series published by Kårhus et al. (1) both patients were taking liraglutide for presenting the comorbidities of overweight and T2DM respectively. Although they remitted the BAD episodes, in the second case it was observed that 7 months after starting treatment, a SeHCAT test was repeated showing $5 \%$ persistence so it would be important to evaluate this situation in detail.

\section{In this context, how can we explain the clinical improvement of GLP-1 in these patients?}

GLP-1 is a human incretin secreted basolaterally by L-cells in response to various stimuli like glucose, aminoacids, BAs and gut microbiome (11). The abundance of GLP-1 secreting L-cells increases towards the distal end of the GI tract (12). Glucose and aminoacids activate small intestinal L-cells whereas colonic L-cells express receptors for BAs. GLP-1 receptors have also been detected in the myenteric plexi in the GI tract which act as a neural regulator of gut contractile activity (13). In the upper part of the GI tract, GLP1 appears to have a mollifying effect on gut function. Using vagal neural pathways, GLP-1 has been shown to delay gastric emptying and small intestinal secretion and motility, whereas in colon it increased colonic transit, also through vagal signaling (11). In a clinical trial in which a synthetic GLP-1 analogue was administered to a mixed group of 99 IBD patients, anti-spasmodic and pain-relieving effects were reported in these patients $v s$. placebo group (14). Gut microbiota should also be studied in patients with BAM since it has been suggested that GI motility is accelerated in intestinal dysbiosis while suppressing the expression of GLP-1 receptor in myenteric neural cells (13). Another association that would be important to investigate in those patients is the role that GLP-1 has on apical sodiumbile transporter (ASBT). It is known that when there is an increase of BAs in postprandial state, FXR inhibits the expression of ASBT and promotes the expression of ileal bile acid-binding protein (IBABP) thus preventing the excessive flux of BAs into the liver and decreasing BAs synthesis by inhibiting CYP7A1 avoiding cytotoxicity (15) (Figure 1). However, the relationship between GLP-1 and ASBT has not been established.

As mentioned before, FXR plays an important role in the pathophysiology of BAM, so it has also been considered as a therapeutic target. In 2018, Hvas et al. (16) published a case report where they described the effectiveness in the use of obeticholic acid in a patient with severe BAM and the antecedent of Crohn's disease. Obeticholic acid is a selective FXR agonist generally used for the treatment of primary biliary cirrhosis. For the management of diarrhea in this patient, the use of BAS was first resorted without obtaining any significant clinical effect, the use of liraglutide was continued without improvement and finally the use of obeticholic acid was administered presenting a clinical improvement within a few days.

In this case the therapeutic failure of liraglutide might be explained by the type of patient, since having the diagnosis of Crohn's disease (BAM type 1) made it impossible to absorb BAs despite the motility effects caused by agonism of GLP-1. The obeticholic acid by agonizing FXR decreased the synthesis of hepatic BAs leading to the remission of BAM by decrease in the BAs pool. This could show that depending on the type of BAM, it would be the best therapeutic tool for their control, despite this, none of the previously mentioned studies have the level of evidence enough to suggest one treatment over another.

In conclusion, BAM is a frequent cause of chronic diarrhea which currently does not have a specific firstline treatment, the pathophysiological mechanisms in which BAM is involved at this time are better understood. However, the treatment for BAM remains a clinical challenge in real life. The results of Kårhus et al.'s study (1) open a new line of research to explore more effective new treatments such as the GLP-1 agonists. In future studies, time and dose-dependent clinical trials are necessary to find out the efficacy, safety and tolerability of this drug. 


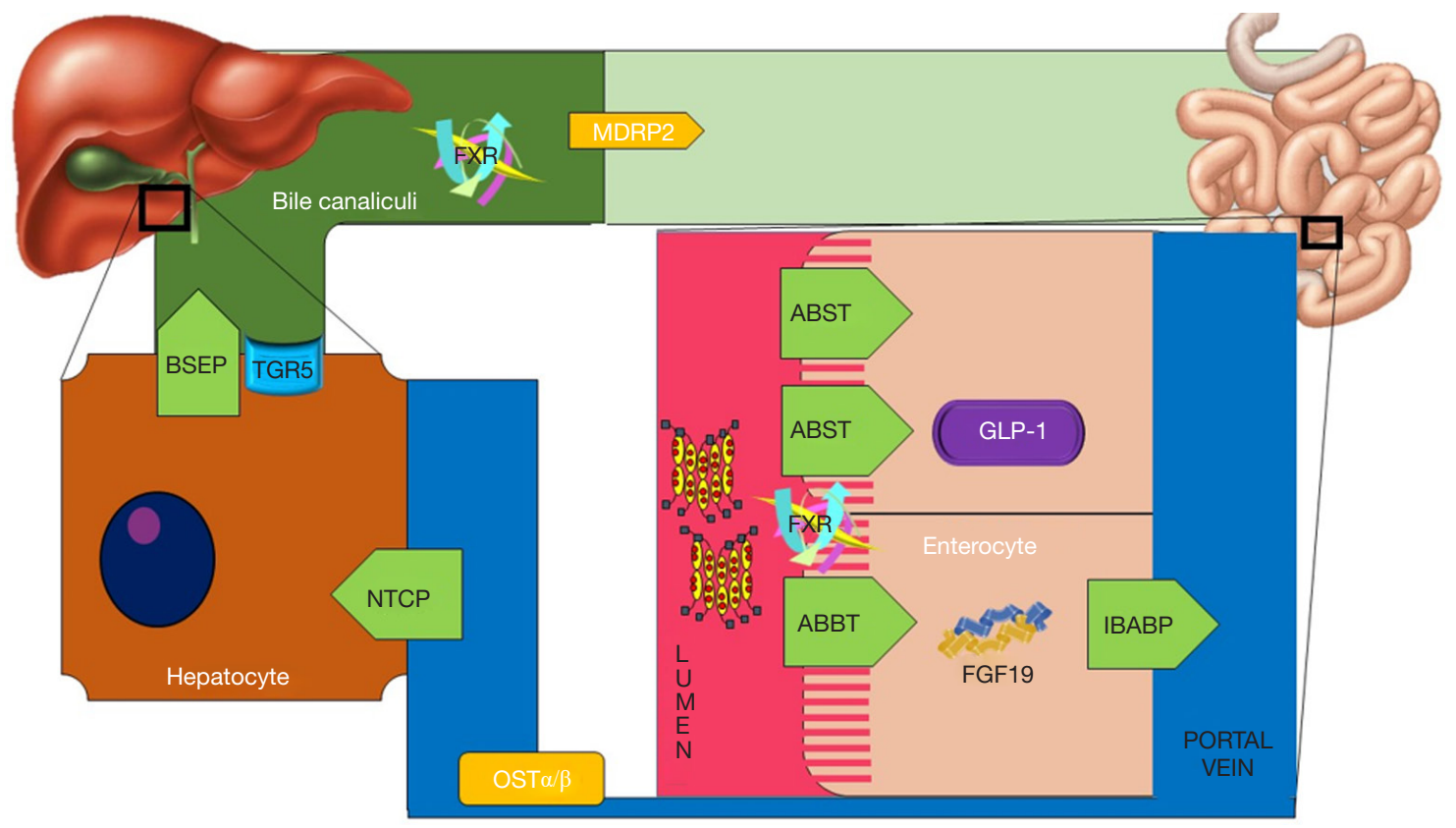

Figure 1 BAs transporters through enterohepatic circulation. In the intestine, specifically distal ileum, BAs are transported within the enterocyte by the action of ASBT, once inside, BAs activate FXR thereby inducing the expression of FGF-19 and by an independent way, BAs activate TGR-5 promoting intestine L cells to release GLP-1. In the enterocyte, the IBABP carriages the BAs to the portal vein, at this point the OST $\alpha$ and OST $\beta$ facilitate their passage to the hepatocyte where NTCP is the primarily responsible for the uptake of BAs from the sinusoids. In the hepatocyte, BAs are conjugated and then transported through the bile canaliculi via BSEP, subsequently MDRP2 delivers conjugates BAs to the gallbladder. This whole process conforms the enterohepatic circulation. However, in BAM, there is an alteration in the BAs pool making them less effective to activate FXR and therefore FGF-19. This causes modifications on BAs transporters, mainly ASBT and IBABP producing a decrease on its absorption in the distal ileum leading to a spillover into the colon. At this point there is a greater activation of TGR-5 increasing the release of GLP-1 by intestinal L cells causing a decrease in gastrointestinal motility. BAs, bile acids; ASBT, apical sodium bile acid transporter; FXR, Farnesoid X receptor; FGF-19, fibroblast growth factor-19; TGR-5, G proteincoupled bile acid receptor 1; GLP-1, glucagon like peptide-1; IBABP, ileal bile acid-binding protein; OST $\alpha / \beta$, organic solute transporter alpha/beta; NTCP, Na+ taurocholate cotransporting polypeptide; BSEP, bile salt export pump; MDRP2, multidrug resistance protein 2; BAM, bile acid malabsorption.

\section{Acknowledgments}

This study was supported in part by a grant from Medica Sur Clinic and Foundation.

\section{Footnote}

Conflicts of Interest: The authors have no conflicts of interest to declare.

Ethical Statement: The authors are accountable for all aspects of the work in ensuring that questions related to the accuracy or integrity of any part of the work are appropriately investigated and resolved.

\section{References}

1. Kårhus ML, Brønden A, Røder ME, et al. Remission of Bile Acid Malabsorption Symptoms Following Treatment With the Glucagon-Like Peptide 1 Receptor Agonist Liraglutide. Gastroenterology 2019;157:569-71.

2. Hofmann AF, Poley JR. Role of bile acid malabsorption in pathogenesis of diarrhea and steatorrhea in patients with ileal resection: $i$. response to cholestyramine or replacement of dietary long chain triglyceride by medium chain triglyceride. Gastroenterology 1972;62:918-34.

3. Vijayvargiya P, Camilleri M. Current Practice in the Diagnosis of Bile Acid Diarrhea. Gastroenterology 2019;156:1233-8. 
4. Camilleri M. Bile Acid diarrhea: prevalence, pathogenesis, and therapy. Gut Liver 2015;9:332-9.

5. Barkun AN, Love J, Gould M, et al. Bile acid malabsorption in chronic diarrhea: pathophysiology and treatment. Can J Gastroenterol 2013;27:653-9.

6. Fani B, Bertani L, Paglianiti I, et al. Pros and Cons of the SeHCAT Test in Bile Acid Diarrhea: A More Appropriate Use of an Old Nuclear Medicine Technique. Gastroenterol Res Pract 2018;2018:2097359.

7. Massafra V, van Mil SWC. Farnesoid X receptor: A "homeostat" for hepatic nutrient metabolism. Biochim Biophys Acta Mol Basis Dis 2018;1864:45-59.

8. Trabelsi MS, Daoudi M, Prawitt J, et al. Farnesoid X receptor inhibits glucagon-like peptide-1 production by enteroendocrine L cells. Nat Commun 2015;6:7629.

9. Chen L, McNulty J, Anderson D, et al. Cholestyramine reverses hyperglycemia and enhances glucose-stimulated glucagon-like peptide 1 release in Zucker diabetic fatty rats. J Pharmacol Exp Ther 2010;334:164-70.

10. Suzuki T, Oba K, Igari Y, et al. Colestimide lowers plasma glucose levels and increases plasma glucagon-like PEPTIDE-1 (7-36) levels in patients with type 2 diabetes mellitus complicated by hypercholesterolemia. J Nippon Med Sch 2007;74:338-43.

Cite this article as: Valencia-Rodríguez A, Aquino-Matus J, Vera-Barajas A, Qi X, Méndez-Sánchez N. New therapeutic options for bile acid malabsorption diarrhea. Ann Transl Med 2019;7(22):695. doi: 10.21037/atm.2019.09.112
11. O'Malley D. Endocrine regulation of gut function - a role for glucagon-like peptide-1 in the pathophysiology of irritable bowel syndrome. Exp Physiol 2019;104:3-10.

12. Steinert RE, Feinle-Bisset C, Asarian L, et al. Ghrelin, CCK, GLP-1, and PYY(3-36): Secretory Controls and Physiological Roles in Eating and Glycemia in Health, Obesity, and After RYGB. Physiol Rev 2017;97:411-63.

13. Yang M, Fukui H, Eda H, et al. Involvement of gut microbiota in association between GLP-1/GLP-1 receptor expression and gastrointestinal motility. Am J Physiol Gastrointest Liver Physiol 2017;312:G367-73.

14. Hellström PM, Hein J, Bytzer P, et al. Clinical trial: the glucagon-like peptide-1 analogue ROSE-010 for management of acute pain in patients with irritable bowel syndrome: a randomized, placebo-controlled, double-blind study. Aliment Pharmacol Ther 2009;29:198-206.

15. Neimark E, Chen F, Li X, et al. Bile acid-induced negative feedback regulation of the human ileal bile acid transporter. Hepatology 2004;40:149-56.

16. Hvas CL, Ott P, Paine P, et al. Obeticholic acid for severe bile acid diarrhea with intestinal failure: A case report and review of the literature. World J Gastroenterol 2018;24:2320-26. 